\title{
Potency of Grain Boundaries as Martensitic Nucleation Sites
}

\author{
K. Tsuzaki, N. Harada and T. Maki \\ Department of Materials Science and Engineering, Kyoto University, Yoshida-honmachi, Sakyo-ku, \\ Kyoto 606-01, Japan
}

\begin{abstract}
The potency of grain boundaries as martensitic nucleation sites has been investigated by measuring the Ms temperatures of bicrystal specimens in an $\mathrm{Fe}-32 \% \mathrm{Ni}$ alloy. The average Ms temperature of single-crystal specimens was $88 \mathrm{~K}$, while that of polycrystalline specimens was $154 \mathrm{~K}$. The free surfaces did not have high potency as nucleation sites, and the higher Ms temperature in the polycrystalline specimens was attributed to the existence of certain kinds of grain boundaries. The Ms temperatures of five kinds of bicrystal specimens having one of annealing twin boundaries and high-angle random boundaries were equivalent to that of the singlecrystal specimens and lower than that of the polycrystalline specimens. These results indicate that both the annealing twin boundaries and the high-angle random boundaries do not have high potency as martensitic nucleation sites, and that the most potent nucleation site which defines the Ms temperature of the polycrystalline specimens is attributed to other kinds of grain boundaries.
\end{abstract}

\section{INTRODUCTION}

The heterogeneous character of martensitic nucleation is now well accepted [1]. However, what kind of lattice defects could be the most favorable nucleation site still remains to be solved. Kajiwara [2] investigated the roles of dislocations and grain boundaries in martensitic nucleation in ferrous alloys. He reported that dislocations themselves do not act as favorable nucleation sites but certain types of grain boundaries would be potent nucleation sites although the exact nature of such boundaries could not be identified. Magee [1] reported that annealing twin boundaries are a potent nucleation site for all types of ferrous martensites. However, martensite plates associated with other kinds of boundaries can be seen in his micrographs where the high potency of annealing twin boundaries was pointed out. It is thus still unclear whether the annealing twin boundaries act as the most potent nucleation site which defines the Ms temperature of a polycrystalline material.

The potency of a certain type of grain boundaries as martensitic nucleation sites can be examined by determining the Ms temperature of bicrystal specimens with a particular boundary and comparing it with the Ms temperatures of polycrystalline and single-crystal specimens. In the present study, the Ms temperatures of bicrystal specimens have been examined to understand how high the potency of annealing twin boundaries and high-angle random boundaries is.

\section{EXPERIMENTAL}

The chemical composition of an $\mathrm{Fe}-32 \% \mathrm{Ni}$ alloy studied was $32.2 \% \mathrm{Ni}, 0.005 \% \mathrm{C}, 0.006 \% \mathrm{Si}$, $0.028 \% \mathrm{Mn}, 0.0009 \% \mathrm{P},<0.001 \% \mathrm{~S}, 0.0071 \% \mathrm{O}, 0.0024 \% \mathrm{~N}$, and balance $\mathrm{Fe}$ (mass $\%$ ). The alloy was prepared by vacuum induction melting and provided as hot-swaged bars or hot-rolled plates. This alloy was remelted to obtain single-crystal and bicrystal specimens. An ingot with a huge grain structure was obtained by the Bridgman method using a high purity alumina crucible with $25 \mathrm{~mm}$ in internal diameter. The ingot was composed of two austenite grains and an annealing twin. As is schematically shown in Fig.1, five kinds of bicrystal specimens were spark-cut to have a $\{111\}$ 


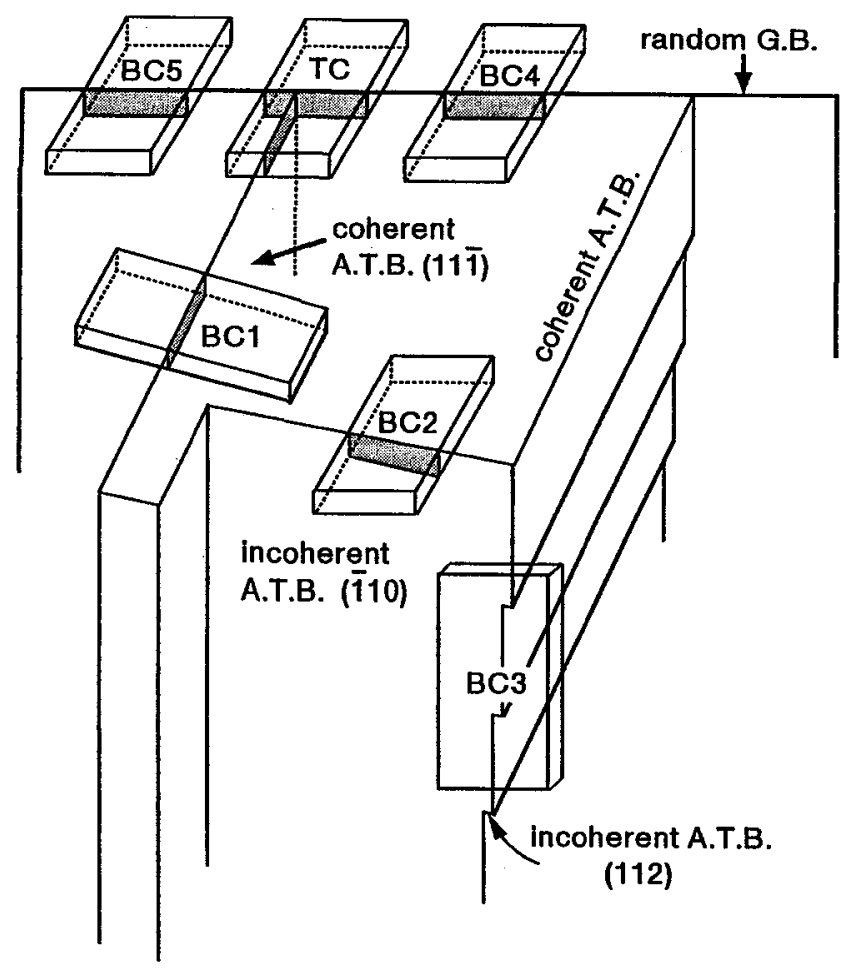

Figure 1: Schematic drawing showing how to cut bicrystal (BC) and tricrystal specimens (TC) from the ingot with a huge grain structure.

The boundary of each bicrystal specimen is a $\{111\}$ coherent twin boundary for $\mathrm{BC} 1, \mathrm{a}\{110\}$ incoherent twin boundary for $\mathrm{BC} 2$, a twin boundary consisting of the steps of $\{111\}$ and $\{112\}$ planes for $\mathrm{BC}$, and one of two kinds of high-angle random boundaries for BC4 and BC5.

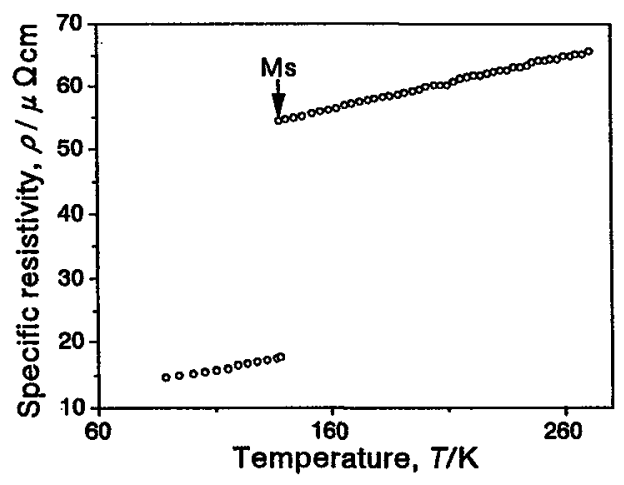

(a)

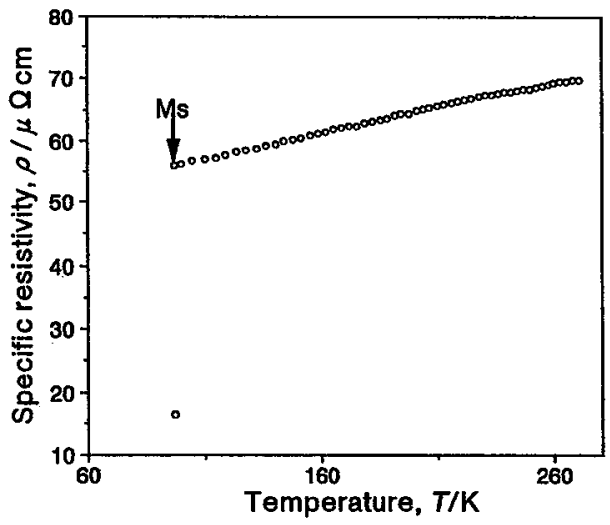

(b)

Figure 2: Electric resistance change with temperature. $\mathrm{Fe}-32 \% \mathrm{Ni}$.

(a) polycrystalline specimen (PC1), Ms: 137K, (b) single-crystal specimen, Ms: $98 \mathrm{~K}$. 
coherent twin boundary (BC1), a $\{110\}$ incoherent twin boundary (BC2), a twin boundary consisting of the steps of $\{111\}$ and $\{112\}$ planes (BC3), and one of two kinds of high-angle random boundaries (BC4 and BC5). Tricystal specimens (TC) were composed of a $\{111\}$ coherent twin boundary and two high-angle random boundaries. Single-crystal specimens were also taken from the same ingot. Polycrystalline specimens were prepared by annealing of the hot-rolled plate with a polycrystalline structure or by applying $20 \%$ cold-rolling and annealing to the single-crystal specimens. The specimen size was $1 \mathrm{~mm} t \times 2 \mathrm{~mm} w \times 6 \mathrm{mml}$.

The orientations of crystals were determined by means of electron channeling pattern (ECP) analysis. In the single-crystal specimens, the longitudinal direction (LD) was $[.140, .900,-.412]$, the transverse direction (TD) was $[.236, .374, .897]$, and the normal direction (ND) was $[-.962, .223,-.160]$. The LD of $\mathrm{BC} 1$ and the TD of $\mathrm{BC} 2$ and $\mathrm{BC} 3$ were close to [11-1]. The high-angle random boundaries (BC4 and $\mathrm{BC5}$ ) were not met for Brandon's criterion up to $\Sigma 51$. For $\mathrm{BC} 4$ the rotation axis was $[1,0.21,3.14]$ and the rotation angle was $34.8 \mathrm{deg}$, while for $\mathrm{BC} 5$ the rotation axis was $[1,-2.11,-1.18]$ and the rotation angle was $48.4 \mathrm{deg}$.

After spark-machining, the specimens for electric resistance measurements were homogenized at $1473 \mathrm{~K}$ for $86.4 \mathrm{ks}$. By this heat treatment, the average linear intercept of austenite grain boundaries became $250 \mu \mathrm{m}$ in the polycrystalline specimens prepared from the hot-rolled plate (PC1) and $600 \mu \mathrm{m}$ in those prepared from single-crystal specimens (PC2), where annealing twin boundaries were not counted. It is noteworthy that in the specimens after homogenizing any segregation of $\mathrm{Ni}$ could not be identified by means of EPMA analysis where the diameter of electron probe was approx. $2 \mu \mathrm{m}$. After polishing surface layers by $50 \mu \mathrm{m}$ and spot-welding of Ni lead wires, the specimens were annealed at $873 \mathrm{k}$ for $1.8 \mathrm{ks}$ to eliminate effects of strain due to polishing and welding. In all the heat treatments, specimens were encapsulated in evacuated silica tubes.

The electric resistance measurements to determine the Ms temperature were carried out by the four points method with a constant current of $500 \mathrm{~mA}$. The specimens were slowly lowered into a $40 \mathrm{~cm}$ height Dewer of which one-fifth was filled with liquid nitrogen. The temperature gradient in the Dewer was $1 \mathrm{~K} / \mathrm{mm}$ at largest. The cooling rate was $5 \mathrm{~K} / \mathrm{min}$ from room temperature to $223 \mathrm{~K}$ and thereafter $1 \mathrm{~K} / \mathrm{min}$.

\section{RESULTS}

All of the present specimens transformed to a large extent in a single burst at the Ms temperature. Examples of electric resistance change measurements are shown in Fig. 2, where the marked decrease in specific resistivity at $\mathrm{Ms}$ is seen. The volume fraction of martensite at Ms, which was obtained by the point counting method, was larger than 0.7 in all the cases.

Figure 3 shows the Ms temperatures of polycrystalline and single-crystal specimens. The Ms temperatures of the two kinds of polycrystalline specimens (PC1 and PC2) are essentially the same although the range of the Ms temperatures is somewhat larger in PC2. The average Ms temperature of the polycrystalline specimens was $154 \mathrm{~K}$. The Ms temperatures of single-crystal specimens are significantly lower than those of the polycrystalline specimens and the highest value is $115 \mathrm{~K}$. Some of the single-crystal specimens did not transform by cooling down to $77 \mathrm{~K}$. Taking $77 \mathrm{~K}$ as the Ms of such specimens, the average Ms temperature of the single-crystal specimens becomes $88 \mathrm{~K}$.

Figure 4 shows the effect of $\mathrm{Ni}$ plating on the Ms temperatures of the single-crystal specimens (SC) and the polycrystalline specimens (PC1). The Ni plating of 15 to $25 \mu \mathrm{m}$ thickness was performed after the final annealing at $873 \mathrm{~K}$. The Ni plating did not suppress the Ms temperatures of both the singlecrystal and polycrystalline specimens. It is thus clear that the free surfaces do not have high potency as nucleation sites and the higher Ms temperature in the polycrystalline specimens is attributed to the existence of certain kinds of grain boundaries which are a potent nucleation site. It is to be noted in Fig. 4 that the range of the Ms temperatures of the single-crystal specimens has become narrow by the $\mathrm{Ni}$ plating, although the reason for this change is not clear at present.

Figure 5 shows the Ms temperatures of the five kinds of bicrystal specimens. In all kinds of the bicrystal specimens with annealing twin boundaries $(\mathrm{BC} 1,2,3)$ and high-angle random boundaries $(B C 4,5)$ the Ms temperatures are lower than $120 \mathrm{~K}$ and comparable to those of the single-crystal 


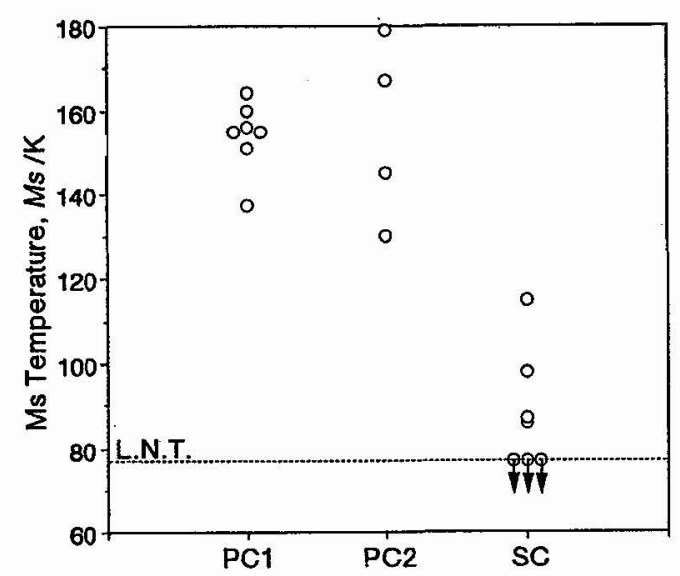

Figure 3: Ms temperatures of polycrystalline specimens (PC1 and PC2) and single-crystal specimens (SC). $\mathrm{Fe}-32 \% \mathrm{Ni}$. The mean liner intercept of austenite grain boundaries is $250 \mu \mathrm{m}$ for PC1 and $600 \mu \mathrm{m}$ for PC2.

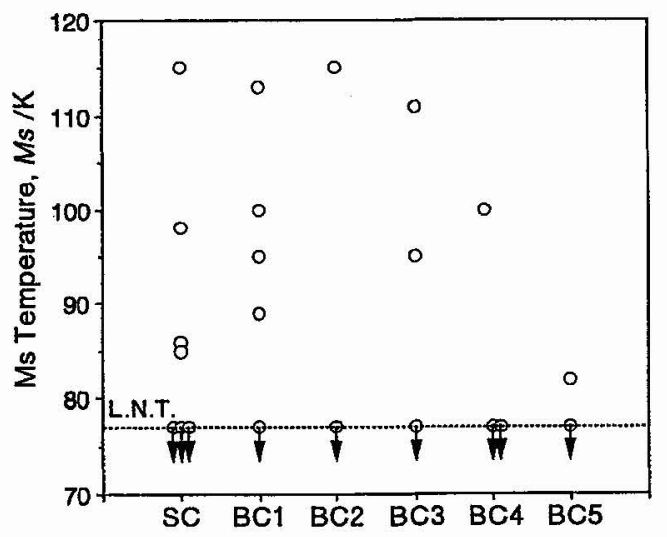

Figure 5: Ms temperatures of single-crystal specimens (SC) and five kinds of bicrystal specimens (BC).

Fe-32\%Ni. Refer Fig.1 for the character of boundaries in the bicrystal specimens.

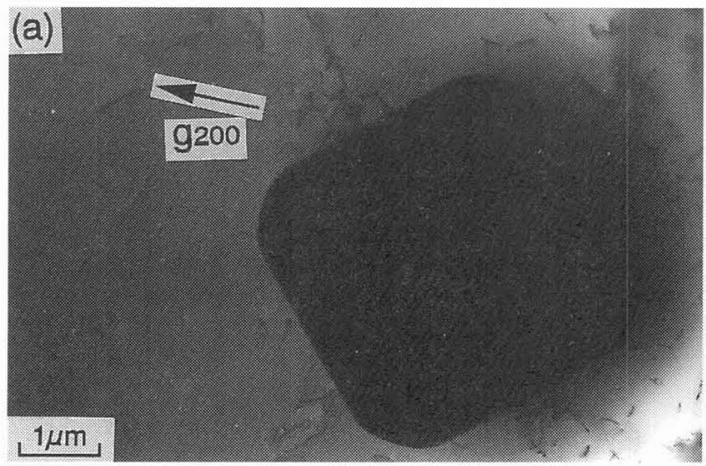

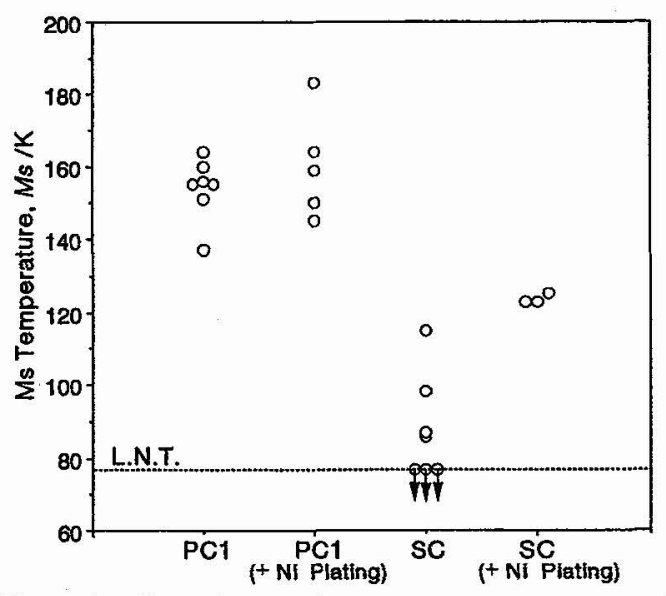

Figure 4: Effect of Ni plating on the Ms temperatures of polycrystalline specimens (PC1) and single-crystal specimens (SC). Fe-32\%Ni.

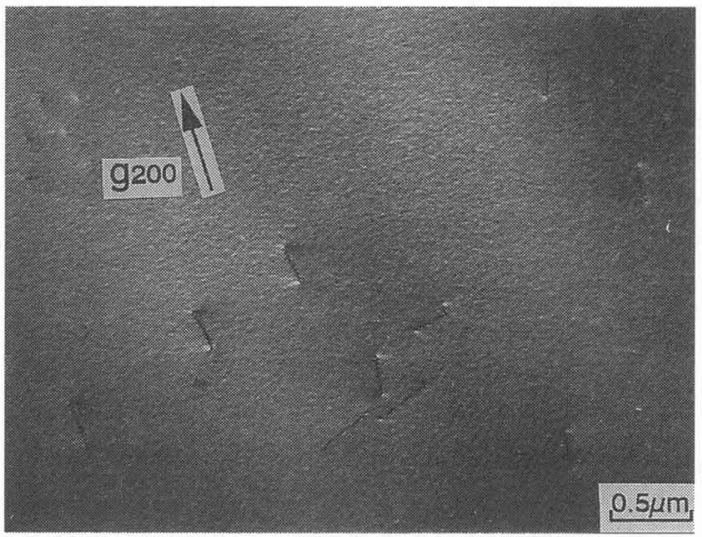

Figure 6: Typical microstructure of the austenite matrix before the transformation where the dislocation density is low. Single-crystal specimen. Fe-32\%Ni. TEM micrograph. Incident beam near [001].

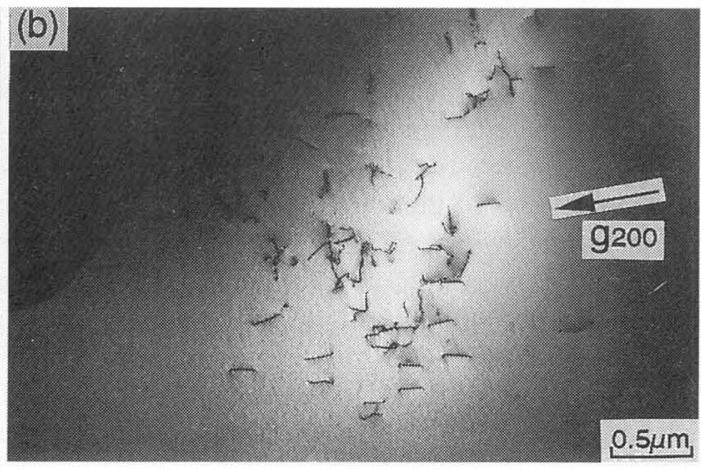

Figure 7: TEM micrograph showing the austenite matrix near a relatively large inclusion where a cluster of dislocations is seen. Single-crystal specimen. Fe-32\%Ni. (b) is a high mag. of (a). Incident beam near [001]. 
specimens. The tricrystal specimens consisting of a $\{111\}$ coherent boundary and two high-angle random boundaries also showed lower Ms temperatures than $120 \mathrm{~K}$. These results indicate that the potency of the annealing twin boundaries and the two high-angle random boundaries is not as high as that of the certain kind of boundaries which defines the Ms temperature of the polycrystalline specimens.

\section{DISCUSSION}

It has been confirmed in the present study that certain kinds of grain boundaries act as the potent nucleation site defining the Ms temperature of a polycrystalline specimen and such a nucleation site is neither the annealing twin boundaries nor the high-angle random boundaries (Figs.3-5). The probability of finding the most potent boundary for martensitic nucleation site can be estimated from the number of grain boundaries in a polycrystalline specimen. Assuming a regular tetrakaidecahedron as the shape of a grain, the average number of grain boundaries without annealing twin boundaries in the present polycrystalline specimens can be estimated to be approx. 2300 for PC1 and 200 for PC2. It was shown in Fig. 3 that PC1 and PC2 exhibit the identical Ms temperature. This identical Ms temperature means that PC2 contains the most potent boundary as PC1 does and 200 grain boundaries are many enough to find it. Thus, the probability of finding the most potent boundary would be $1 / 200$ (one per 200 grain boundaries) or higher.

The bicrystal specimens containing one of annealing twin boundaries and high-angle random boundaries showed the equivalent Ms temperatures to those of the single-crystal specimens (Fig. 5). This same Ms temperature suggests that the same kind of nucleation sites acted as the initial nucleation site in both the single-crystal and bicrystal specimens and neither the annealing twin boundaries nor the high-angle random boundaries were the initial nucleation site. The experiments with the Ni plated specimens clearly denied the possibility that the free surfaces act as the initial nucleation site (Fig. 4), and thereby other defects must act for the first nucleation event. Although the exact initial nucleation site in the bicrystal and single-crystal specimens could not be identified by metallography due to a burst transformation, it is noteworthy that there are some non-metallic inclusions in the present specimens. A cluster of dislocations was seen near inclusions, while a low density of dislocations was observed in the austenite matrix irrespective of single-crystal and bicrystal specimens (Fig. 6). Figure 7 is a TEM micrograph showing the austenite matrix near a relatively large inclusion. A number of dislocations is seen near the inclusion. Such inclusions exist in both the single-crystal and bicrystal specimens with the same probability, note that these specimens in addition to $\mathrm{PC} 2$ were prepared from the same ingot. It is thus possible that these dislocations near inclusions or inclusion interfaces act as the initial nucleation site, resulting in almost identical Ms temperature in the bicrystal and single-crystal specimens. This argument deduces a conclusion that the potency of the annealing twin boundaries and the high-angle random boundaries is lower than that of inclusion interfaces or a cluster of dislocations.

The low potency of annealing twin boundaries observed in the present study is contrary to the result reported by Magee [1] where the high potency of twin boundaries was demonstrated. Due to the low energy of coherent twin boundaries, he assumed that incoherent steps or ledges on these boundaries are the actual nucleation site. However, he could not obtain any direct evidence for this idea by extensive cold-stage experiments on untransformed thin films. It was thereby presumed that the actual nucleation site is still more specialized and an example possibility would be an inclusion at an incoherent twin boundary segment. In the present bicrystal specimens, any inclusion associated with incoherent twin boundary segments could not be identified. It is thus likely that even incoherent steps on twin boundaries do not act as a potent nucleation site when the steps are free from inclusions.

Magee [1] and Christian [3] proposed that a highly strained region acts as a potent site for martensitic nucleation, based on the idea that the strain field of a martensite particle or embryo interacts with the strain field of the nucleation site so that the free energy barrier for the nucleation decreases. According to this idea of a highly strained region for martensitic nucleation sites, the low potency of high-angle random boundaries observed in the present study is easily understood, because these boundaries do not possess long-range stress fields [4].

Olson and Cohen [5] have proposed that the first step of martensitic nucleation is faulting on planes of closest packing. The stacking faults develop from existing lattice defects and these groups of faults 
become unstable and undergo a spontaneous transformation during cooling. They considered that such defects should consist of dislocations with appropriate numbers and spacing and should possess longrange stress fields. The symmetric tilt boundaries with the rotation axis of $\langle 112\rangle$ have a similar dislocation configuration to that of the dislocation dissociation model [5]. It may be thus possible that the most potent nucleation site defining the Ms temperature of a polycrystalline specimen is such tilt boundaries.

Krauss et al. [6] reported that the Ms temperature of single-crystal small particles of an $\mathrm{Fe}-30 \mathrm{at} \% \mathrm{Ni}$ alloy is markedly suppressed by $\mathrm{Ni}$ coating and concluded that free surfaces are effective nucleation sites. However, the Ni coating did not suppress the Ms temperature of the present single-crystal specimens. This discrepancy can be accounted for by the size of the samples investigated. Since the single-crystal particles studied by Krauss et al. is small, 48 to $114 \mu \mathrm{m}$ in diameter, the probability of finding defects such as inclusions within a particle sample is low and the free surface would be the most potent nucleation site in a given sample. In such a case, the elimination of free surfaces by Ni coating can lower the Ms temperature. On the other hand, the present single-crystal specimens are relatively large, and hence they contain a number of inclusions. That is, the free surface is not the most potent nucleation site in the sample. We consider that the effect of Ni coating on the Ms temperature depends on whether the free surface is the most potent nucleation site in a given sample and the effect would be siginificant in small samples.

\section{CONCLUSIONS}

The Ms temperatures of single-crystal, bicrystal and polycrystalline specimens in an $\mathrm{Fe}-32 \% \mathrm{Ni}$ alloy has been examined to understand the potency of annealing twin boundaries and high-angle random boundaries as martensitic nucleation sites. The main results obtained are as follows.

1. The Ms temperature of single-crystal specimens is $88 \mathrm{~K}$, while that of polycrystalline specimens is 154K. The Ni plating does not suppress the Ms temperature. These results indicate that the potency of free surfaces is not high and certain kinds of grain boundaries act as a potent nucleation site. The probability of finding the most potent boundary can be estimated to be one per 200 grain boundaries $(1 / 200)$ or higher.

2. The Ms temperatures of five kinds of bicrystal specimens having one of annealing twin boundaries and high-angle random boundaries are equivalent to that of the single-crystal specimens and lower than that of the polycrystalline specimens, indicating that the annealing twin boundaries and high-angle random boundaries do not have high potency and the most potent nucleation site which defines the Ms temperature of the polycrystalline specimens is attributed to other kinds of grain boundaries.

3. The sites acted for the first nucleation event in both the bicrystal and single-crystal specimens are inclusion interfaces or a cluster of dislocations formed near inclusions, and the potency of the annealing twin boundaries and the high-angle random boundaries is lower than that of those sites associated with inclusions.

Acknowledgments: The authors would like to express their sincere thanks to Dr. A. Otsuki and Dr. T. Furuhara (Kyoto University) for important advice.

\section{References}

[1] C.M.Magee, Phase Transformations (ASM, Metals Park, Ohio, 1969) pp.115-156.

[2] S.Kajiwara, Met. Trans. A, 17A (1986) 1693-1702.

[3] J.W.Christian, The Mechanism of Phase Transformations in Crystalline Solids (Institute of Metals, London, 1969) pp.129-142.

[4] J.P.Hirth and J.Lothe, Theory of Dislocations, 2nd Ed. (John Wiley \& Sons, New York, 1982) pp.697-750.

[5] G.B.Olson and M.Cohen, Metall. Trans. A, 7A (1976) 1897-1904, 1905-1914, 1915-1923.

[6] W.Krauss, S.K.Pabi and H.Gleiter, Acta metall, 37 (1989) 25-30. 